\title{
Primordial Nucleosynthesis: Constraints on the Birth of the Universe
}

\author{
Grant Mathews $^{1, \star}$, Motohiko Kusakabe ${ }^{1,2}$, Mayukh Gangopadhyay ${ }^{1,3}$, Toshitaka Kajino ${ }^{2,4,5}$, and \\ Nishanth Sasankan ${ }^{1}$ \\ ${ }^{1}$ Center for Astrophysics, Department of Physics, University of Notre Dame, Notre Dame, IN 46556 USA \\ ${ }^{2}$ School of Physics and Nuclear Energy Engineering, and International Research Center for Big-Bang Cos- \\ mology and Element Genesis, Beihang University, Beijing 100191, P.R. China \\ ${ }^{3}$ Theory Division, Saha Institute of Nuclear Physics, 1/AF, Bidhannagar, Kolkata- 64, India \\ ${ }^{4}$ National Astronomical Observatory of Japan 2-21-1 Osawa, Mitaka, Tokyo, 181-8588 Japan \\ ${ }^{5}$ Department of Astronomy, Graduate School of Science, The University of Tokyo, 7-3-1 Hongo, Bunkyo-ku, \\ Tokyo, 113-0033 Japan
}

\begin{abstract}
We review the basic elements of big bang nucleosythesis (BBN) and how a comparison of predicted light-element abundances with observations constrains physics of the radiation-dominated epoch. We then summarize some applications of BBN and the cosmic microwave background (CMB) to constrain the first moments of the birth of the universe. In particular, we discuss how the existence of higher dimensions impacts the cosmic expansion through the projection of curvature from the higher dimension in the "dark radiation" term. We summarize current constraints from BBN and the CMB on this brane-world dark radiation term. At the same time, the existence of extra dimensions during the earlier inflation impacts the tensor to scalar ratio and the running spectral index as measured in the CMB. We summarize how the constraints on inflation shift when embedded in higher dimensions. Finally, one expects that the universe was born out of a complicated multiverse landscape near the Planck time. In these moments the energy scale of superstrings was obtainable during the early moments of chaotic inflation. We summarize the quest for cosmological evidence of the birth of space-time out of the string theory landscape. We will explore the possibility that a superstring excitations may have made itself known via a coupling to the field of inflation. This may have left an imprint of "dips" in the power spectrum of temperature fluctuations in the cosmic microwave background. The identification of this particle as a superstring is possible because there may be evidence for different oscillator states of the same superstring that appear on different scales on the sky. It will be shown that from this imprint one can deduce the mass, number of oscillations, and coupling constant for the superstring. Although the evidence is marginal, this may constitute the first observation of a superstring in Nature.
\end{abstract}

\section{Introduction}

We are at a unique period in the history of the human understanding of the cosmos. For the first time, we have a clear picture of what the universe is comprised of, how long it has been in existence, and

^e-mail: gmathews@nd.edu 
how it will evolve in the future. This knowledge is the culmination of investigations via a number of cosmological probes including supernovae, observations of the large scale distribution of galaxies and the inter-galactic medium, analysis of the cosmic microwave background, observations of the first stars of the early universe, and studies of the nucleosynthesis of the elements in the first few moments of cosmic expansion. Here, we review a number of outstanding questions in contemporary cosmology and highlight the input that big bang nucleosynthesis (BBN) and the Cosmic Microwave Background provides toward answering them.

\subsection{Elementary Dynamics of Space-time}

If one adopts the notion that the universe is homogeneous and isotropic on a large enough scale, then space-time intervals are given by the Lemaitre-Friedmann-Robertson-Walker metric,

$$
d s^{2}=-d t^{2}+a(t)^{2}\left[\frac{d r^{2}}{1-k r^{2}}+r^{2} d \theta^{2}+r^{2} \sin ^{2} \theta d \phi^{2}\right],
$$

where now $a(t)$ is the dimensionless scale factor and $k$ is the curvature parameter and we adopt units of $\hbar=c=1$. For this metric, the evolution of the early universe is simply given by the Friedmann equation which describes the the Hubble parameter $\mathrm{H}$ in terms of densities $\rho$, curvature $k$, the cosmological constant $\Lambda$, and the cosmic scale factor $a$ :

$$
\left(\frac{\dot{a}}{a}\right)^{2} \equiv H^{2}=\frac{8}{3} \pi G \rho-\frac{k}{a^{2}}+\frac{\Lambda}{3}=H_{0}^{2}\left[\frac{\Omega_{\gamma}}{a^{4}}+\frac{\Omega_{m}}{a^{3}}+\frac{\Omega_{k}}{a^{2}}+\Omega_{\Lambda}\right],
$$

where $H_{0}=67.74 \pm 0.46 \mathrm{~km} \mathrm{sec}^{-1} \mathrm{Mpc}^{-1}$ is the present value [1] of the Hubble parameter. One can then define the various closure contributions from relativistic particles, nonrelativistic matter, curvature, and dark energy:

$$
\Omega_{\gamma}=8 \pi G \rho_{\gamma} /\left(3 H_{0}^{2}\right), \Omega_{m}=8 \pi G \rho_{m} /\left(3 H_{0}^{2}\right), \Omega_{k}=k /\left(a^{2} H_{0}^{2}\right), \Omega_{\Lambda}=\Lambda /\left(3 H_{0}^{2}\right) .
$$

Based upon the Planck analysis [1] then one can deduce that the contribution to closure from baryonic matter is $\Omega_{b}=0.0486 \pm 0.011$. The total matter content, however is $\Omega_{m}=0.3089 \pm$ 0.0062. This implies that a much larger fraction of the universe is made of a completely unknown component of "cold dark matter," $\Omega_{c}=0.260 \pm 0.006$. Even more surprising is that the universe is predominantly made of a completely exotic form of mass energy, i.e. dark energy, denoted as, $\Omega_{\Lambda}=$ $0.691 \pm 0.006$ for the case of a cosmological constant. In addition to these, there is presently an almost negligible mass-energy contribution from relativistic photons and neutrinos which we designate as $\Omega_{\gamma}=\rho_{\gamma} / \rho_{c}=5.46(19) \times 10^{-5}$. The early universe includes the Planck epoch, the birth of space-time, inflation, reheating, a variety of cosmic phase transitions (e.g. supersymmetry breaking, baryogenesis, the electroweak transition, and the QCD transition), the epoch of big bang nucleosynthesis, and the production of the cosmic microwave background (CMB). The universe remains radiation dominated for most of the big bang, so that the radiation $\left[\Omega_{\gamma}\right.$ term in Eq. (2)] is important during BBN. There are, however, interesting variants of big bang cosmology where this is not the case as described below.

The precision with which these parameters are now known is much better than a decade ago, however, one must keep in mind that $t$ these parameters are based upon the simplest possible $\Lambda \mathrm{CDM}$ cosmology, and the analysis must be redone for more complicated cosmologies such as those described below.

The only direct probe of the radiation dominated epoch is the yield of light elements from BBN in the temperature regime from $10^{8}$ to $10^{10} \mathrm{~K}$ and times of about 1 to $10^{3} \mathrm{sec}$ into the big bang. The only other probe is the spectrum of temperature fluctuations in the $\mathrm{CMB}$ which contains information of 
the first quantum fluctuations in the universe, and the details of the distribution and evolution of dark matter, baryonic matter, photons and electrons near the time of the surface of photon last scattering (about $2.8 \times 10^{5}$ yr into the big bang).

One of the most powerful aspects of BBN is the simplicity [2-7] of the equations. For the most part one can assume thermodynamic equilibrium of all species present. The only non-equilibrium processes are the thermonuclear reactions themselves. For all times relevant to standard big-bang nucleosynthesis, one can ignore curvature and dark energy. Hence, the Friedmann equation is just:

$$
\left(\frac{\dot{a}}{a}\right)=\sqrt{\frac{8}{3} \pi G \rho_{\gamma}}=H_{0} \frac{\sqrt{\Omega_{\gamma}}}{a^{2}} \approx 1.13 T_{\mathrm{MeV}}^{2} \mathrm{sec}^{-1} .
$$

Also, at the time of BBN the timescale for Compton scattering is short. Hence, the number density of particles of type $i$ with momenta between $p$ and $p+d p$ is simply given by Fermi-Dirac or BoseEinstein distributions,

$$
n_{i}(p) d p=\frac{g_{i}}{2 \pi^{2}} p^{2}\left[\exp \left(\frac{E_{i}(p)-\mu_{i}}{k T} \pm 1\right]^{-1} d p\right.
$$

where $E_{i}(p)$ is the energy of the particle, the \pm sign is negative for bosons and positive for fermions, while $g_{i}$ is the number of degenerate spin states of the particle (e.g. $g=1$ for neutral massless leptons, and $g_{i}=2$ for charged leptons and photons).

Integrating the product $E_{i}(p) n_{i}(p)$ over all momenta gives the energy density for particle $i$. In the standard big bang, with all chemical potentials $\mu_{i}$ set to zero, the total mass-energy density of the universe at the epoch of nucleosynthesis is given by

$$
\rho=\rho_{\gamma}+\rho_{v_{i}}+\rho_{i},
$$

where $\rho_{\gamma}, \rho_{\gamma_{i}}$, and $\rho_{i}$ are the energy densities due to photons, neutrinos, and charged leptons, respectively (including antiparticles). Note that during BBN, the baryons contribute negligibly to the total mass energy density. At the temperatures of primordial nucleosynthesis only electrons (and positrons) contribute to the charged leptons. The contribution from neutrinos is given by (assuming only relativistic particles)

$$
\rho_{v_{i}}=\int p\left[n_{v_{i}}(p)+n_{\bar{v}_{i}}(p)\right] d p=\frac{7}{8} \frac{\pi^{2}}{15}\left(T_{v_{i}}\right)^{4}
$$

where $T_{v_{i}}$ is the neutrino temperature and here and for the rest of the manuscript we adopt natural units, i.e. $k=c=\hbar=1$. The energy densities from photons $\rho_{\gamma}$ and relativistic charged leptons $\rho_{i}$ are given by

$$
\rho_{\gamma}=\frac{4}{7} \rho_{i}=\frac{\pi^{2}}{15}\left(T_{\gamma}\right)^{4}
$$

where $T_{\gamma}$ is the photon temperature (henceforth we absorb the Boltzmann constant $\mathrm{k}$ into the temperature). For temperatures above $1 \mathrm{MeV}$, the neutrinos and photons are in thermal equilibrium $\left(T_{\gamma}=T_{v_{i}}=T\right)$.

The Friedmann equation plus the fact that temperature decreases inversely with the scale factor, can be used to derive the relation between temperature and time during BBN.

$$
T \approx 1.4 g_{\mathrm{eff}}^{-1 / 4}\left(\frac{t}{1 \mathrm{sec}}\right)^{-1 / 2} \mathrm{MeV},
$$

where $g_{\mathrm{eff}}(T)$ is the effective number of relativistic degrees of freedom in bosons and fermions,

$$
g_{\text {eff }}(T)=\sum_{\text {Bose }} g_{\text {Bose }}+\frac{7}{8} \sum_{\text {Fermi }} g_{\text {Fermi }} .
$$


This completes the equations of the basic thermodynamics. The nuclear reactions, however, must be followed in detail as they fall out of equilibrium. For nuclide $i$ undergoing reactions of the type $i+j \leftrightarrow l+k$ one can write:

$$
\frac{d Y_{i}}{d t}=\sum_{i, j, k} N_{i}\left(\frac{Y_{l}^{N_{l}} Y_{k}^{N_{k}}}{N_{l} ! N_{k} !} n_{k}\left\langle\sigma_{l k} v\right\rangle-\frac{Y_{i}^{N_{i}} Y_{j}^{N_{j}}}{N_{i} ! N_{j} !} n_{j}\left\langle\sigma_{i j} v\right\rangle\right)
$$

where $Y_{i}=X_{i} / A_{i}$ is the abundance fraction, $N_{i}$ is the number of reacting identical particles, $n_{i}$ is the number density of nucleus $i$ and $\left\langle\sigma_{i j} v\right\rangle$ denoted the maxwellian averaged reaction cross section,

$$
\left\langle\sigma_{i j} v\right\rangle=\sqrt{\frac{8}{\pi \mu_{i j}}}(T)^{-\frac{3}{2}} \int_{0}^{\infty} \sigma_{i j}(E) \exp [-E / T] E d E
$$

where $\mu_{i j}=m_{i} m_{j} /\left(m_{i}+m_{j}\right)$ is the reduced mass of the reacting system. Once the forward reaction rate is known, the reverse reaction rate can be deduced from an application (cf. Ref. [8]) of the principle of detailed balance. For a two body reaction one can write:

$$
\langle\sigma v\rangle_{l k}=\frac{g_{i} g_{j}}{g_{l} g_{k}\left(1+\delta_{12}\right)}\left(\frac{\mu_{i j}}{\mu_{k l}}\right)^{3 / 2}\left\langle\sigma_{12} v\right\rangle \exp [-Q / T],
$$

where $Q$ is the energy released in the reaction. The reaction rates relevant to $\mathrm{BBN}$ have been conveniently tabularized in analytic form in several sources [9-11]. These rates are a crucial ingredient to BBN calculations.

In all there are only 16 reactions of significance during BBN. [5, 6, 12]. In order to be useful as a cosmological constraint one must know relevant nuclear reaction rates to very high precision $(\sim 1 \%)$. Fortunately, unlike in stars, the energies at which these reactions occur in the early universe are directly accessible in laboratory experiments. Although considerable progress has been made $[6,12-16]$ toward quantifying and reducing uncertainties in the relevant rates, improved reaction rates are still needed for the neutron life time [17, 18], the ${ }^{2} \mathrm{H}(p, \gamma){ }^{3} \mathrm{He},{ }^{2} \mathrm{H}(d, n){ }^{3} \mathrm{He},{ }^{3} \mathrm{He}(d, p){ }^{3} \mathrm{He}$, ${ }^{3} \mathrm{He}(\alpha, \gamma){ }^{7} \mathrm{Be}$, and ${ }^{7} \mathrm{Be}(n, \alpha){ }^{4} \mathrm{He}$ reactions.

\section{Light Element Abundances}

One of the powers of standard-homogeneous BBN is that once the reaction rates are known, all of the light element abundances are determined in terms of the single parameter $\eta$ (or $\eta_{10}$ defined as the baryon-to-photon ratio in units of $10^{10}$ ). The crucial test of the standard BBN is, therefore, whether the independently determined value of $\eta_{10}$ from fits to the CMB reproduces all of the observed primordial abundances. There are new constraints on the primordial helium abundance [19]. The best abundance constraints for this review are then adopted from Ref. [6] as follows:

\subsection{Deuterium}

Deuterium is best measured in the spectra of narrow-line Lyman- $\alpha$ absorption systems in the foreground of high redshift QSOs. Moreover, i one restricts the data to the six well resolved systems for which there are multiple Lyman- $\alpha$ lines $[6,20]$, one obtains the presently adopted value [6] of $\mathrm{D} / \mathrm{H}=2.53 \pm 0.04 \times 10^{-5}$. 


\section{$2.2{ }^{3} \mathrm{He}$}

The abundance of ${ }^{3} \mathrm{He}$ is difficult to detect and the only measurement is from a single Galactic HII region [21] based upon the $8.665 \mathrm{GHz}$ hyperfine transition of ${ }^{3} \mathrm{He}^{+}$. A plateau with a relatively large dispersion with respect to metallicity was found at a level of ${ }^{3} \mathrm{He} / \mathrm{H}=(1.90 \pm 0.6) \times 10^{-5}$. It is not yet understood, however, whether ${ }^{3} \mathrm{He}$ has increased or decreased through the course of stellar and galactic evolution [22, 23]. Fortunately, one can avoid the ambiguity in galactic ${ }^{3} \mathrm{He}$ production by making use of the fact that the sum of $\left(\mathrm{D}+{ }^{3} \mathrm{He}\right) / \mathrm{H}$ is largely unaffected by stellar processing. This leads to a reasonable $2 \sigma$ upper limit $[5,13]$ of ${ }^{3} \mathrm{He} / \mathrm{H}<1.7 \times 10^{-5}$.

\section{$2.3{ }^{4} \mathrm{He}$}

The primordial ${ }^{4} \mathrm{He}$ abundance, $\mathrm{Yp}$ is best determined from HII regions in metal poor irregular galaxies extrapolated to zero metallicity. In [19] it was demonstrated that updated emissivities and the neutral hydrogen corrections generally increase the inferred abundance, while the correlated uncertainties increase the uncertainty in the final extracted helium abundance. This leads to: $Y_{p}=0.2449 \pm 0.0040$.

\section{$2.4^{7} \mathbf{L i}$}

The primordial abundance of ${ }^{7} \mathrm{Li}$ is best determined from old metal-poor halo stars at temperatures corresponding to the Spite plateau (see Refs. [5, 6, 13] and Refs. therein). There is, however, an uncertainty in this determination due to the fact that the surface lithium in these stars may have experienced gradual depletion due to mixing with the higher temperature stellar interiors over the stellar lifetime. The best current limits are from Ref. [6]: ${ }^{7} \mathrm{Li} / \mathrm{H}=(1.6 \pm 0.3) \times 10^{-10}$.

\section{Comparison of BBN with Observed abundances}

Ultimately, the value of BBN is in the detailed comparison between the primordial abundances inferred from observation and the predictions based upon the BBN calculation. There is, however, one glaring problem that remains. The calculated and observed ${ }^{7} \mathrm{Li} / \mathrm{H}$ ratio differ by about a factor of 3. This is known as "the lithium problem." A number of recent papers have addressed this problem $[13,14,25-28]$. At present it is not yet known if this discrepancy derives from a destruction of Lithium on the old stars used to deduce the primordial Lithium abundance, or if it requires exotic new physics in the early universe [13, 14, 25-27], or even a modification of the particle statistics in BBN itself [28]. For the remainder of this introduction we will adopt the premise that this disagreement may indicate new physics in the early universe. It is important then to address what are the key questions in modern cosmology and how might BBN address these questions.

\subsection{Is there Evidence for Large Extra Dimensions?}

In one form of the low-energy limit to M-theory [29] the universe can be represented by two 10dimensional manifolds separated by a large extra dimension. It is possible that the extra dimension could manifest itself on the dynamics of the universe and BBN [30, 31]. For example, in a RandallSundrum II [32] brane-world cosmology, the cosmic expansion for a 3-space embedded in a higher dimensional space can be written $[30,31]$ as

$$
\left(\frac{\dot{a}}{a}\right)^{2}=\frac{8}{3} \pi G \rho-\frac{k}{a^{2}}+\frac{\Lambda}{3}+\frac{\kappa_{5}^{4}}{36} \rho^{2}+\frac{\mu}{a^{4}},
$$




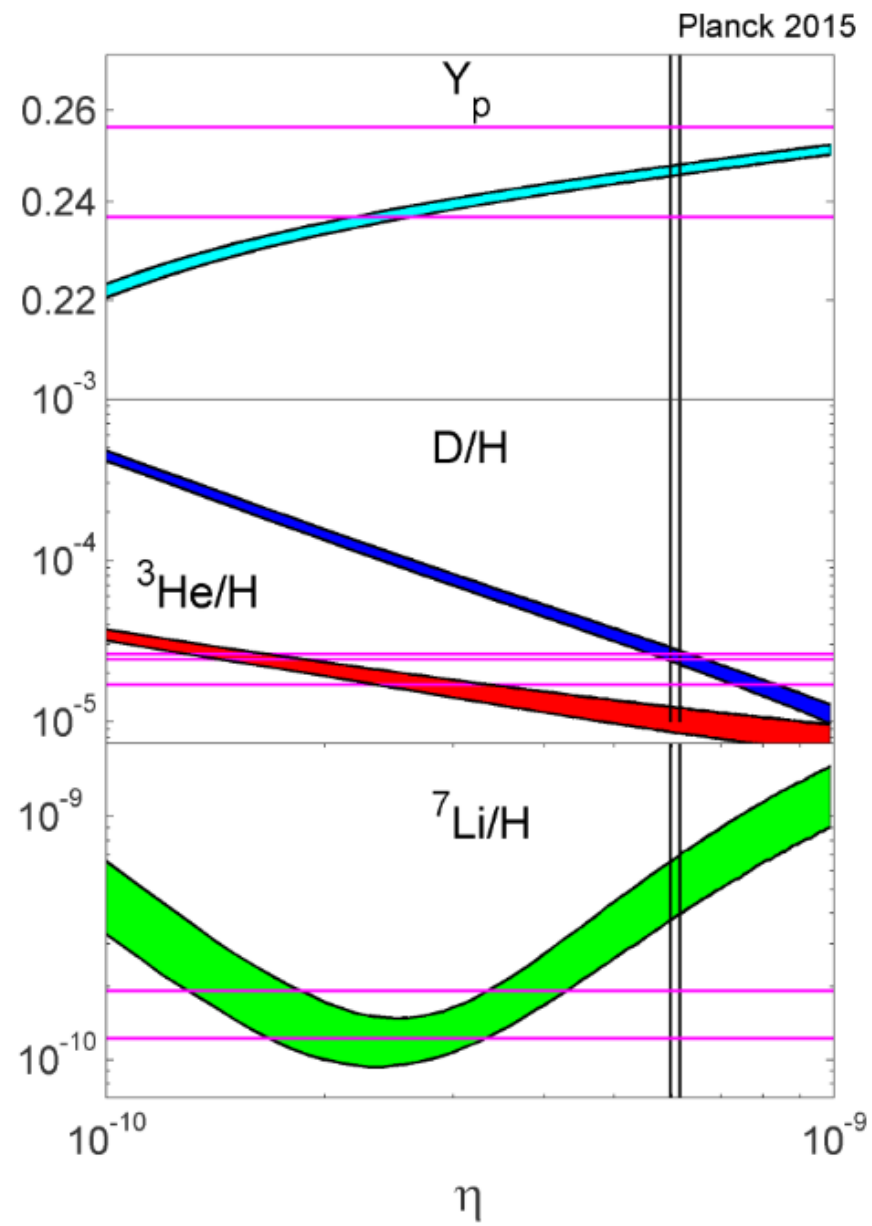

Figure 1. BBN abundances as a function of the baryon to photon ratio. Shaded bands correspond to the $2 \sigma$ ( $95 \%$ C.L.) uncertainties deduced in [12]. Horizontal lines show range of the uncertainties in the primordial abundances inferred in [12]. Vertical lines indicate the value of $\eta$ deduced in the Planck analysis [1].

where the four-dimensional gravitational constant $G_{N}$ is related to $\kappa_{5}$ the five-dimensional gravitational constant, i.e. $G_{N} \kappa_{5}^{4} \lambda / 48 \pi$, with $\lambda$ the intrinsic tension of the brane. The fourth term arises from the imposition of a junction condition for the scale factor on the surface of the brane, and is not likely to be significant during BBN. However it is significant during inflation [33] as described below. The fifth term, however, scales just like radiation with a constant $\mu$ and is called the dark radiation. Hence, it can contribute during BBN. Its magnitude and sign derives from the projection of curvature in higher dimensions onto four-dimensional space-time. Because this dark radiation scales as $a^{-4}$ it can affect both $\mathrm{BBN}$ and the $\mathrm{CMB}$. It can significantly alter $[30,31]$ the fit to $\mathrm{BBN}$ abundances and the $\mathrm{CMB}$, and hence can be constrained. In [31] it was shown that the newest light element abundance constraints and reaction rates significantly limits the possibility of brane-world dark radiation. 


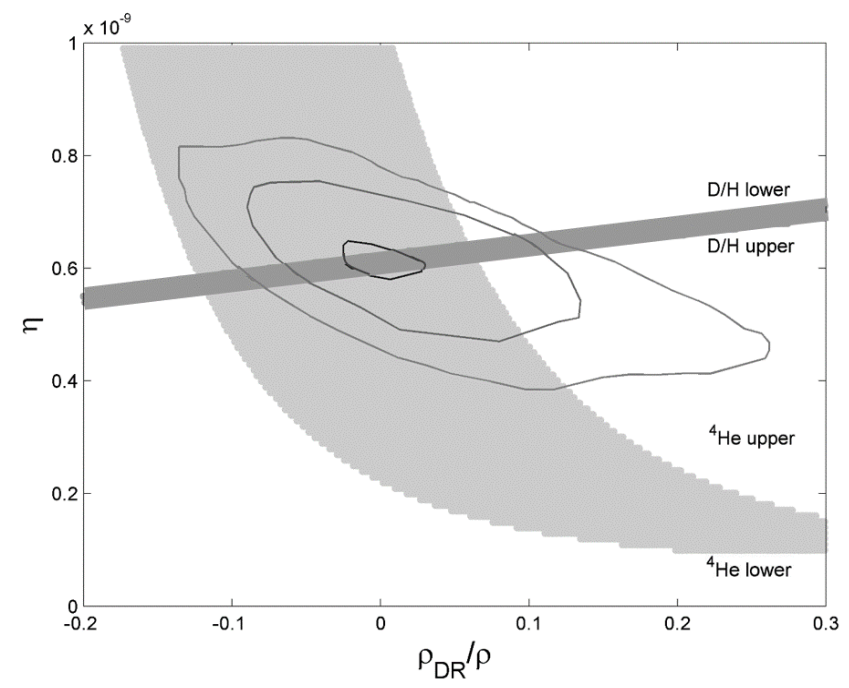

Figure 2. Constraints on dark radiation in the $\rho_{\mathrm{DR}} / \rho(10 \mathrm{MeV})$ vs. $\eta$ plane from Ref. [31]. Contour lines show the 1,2 , and $3 \sigma$ confidence limits based upon our fits to the CMB power spectrum. Dark shaded lines show the constraints from the primordial deuterium abundance as described in the text. The light shaded region shows the $Y_{p}$ constraint.

Figure 2 shows the combined constraints on $\eta$ vs. dark radiation based upon our fits to both BBN and the $\mathrm{CMB}$ power spectrum. Here, the fraction of dark radiation is normalized relative to the energy density $\rho(10 \mathrm{MeV})$ at a big bang temperature of $10 \mathrm{MeV}$. The contour lines on Figure 2 show the CMB 1, 2, and $3 \sigma$ confidence limits in the $\eta$ vs. dark radiation plane. The shaded regions show the $\mathrm{BBN} Y_{p}$ and $\mathrm{D} / \mathrm{H}$ constraints as labeled.

The best fit concordance shown in Figure 2 is consistent with no dark radiation although in the BBN analysis there is a slight preference for negative dark radiation. A similar result was found in the previous analysis of Ref. [30]. However, the magnitude of any dark radiation is much more constrained in the present analysis.

Similarly, in [33] we have analyzed the Randal Sundrum brane-world inflation scenario in the context of the latest CMB constraints from Planck. The simplest explanation for the fact that the universe is so nearly at today $\left(\Omega_{0}=1.000 \pm 0.005\right.$ Ref. [1]) and the near isotropy of CMB is to conclude that the universe has gone through an epoch rapid inflation.[34] The simplest view is that some vacuum energy $V(\phi)$ drives inflation due to the existence of a self-interacting scalar field $\phi$. That is, the energy density of the cosmic fluid in the early universe includes a dominant contribution from the evolution of a scalar field. The mass energy density for a scalar field can be deduced from the Klein-Gordon Equation $\rho(\phi)=\frac{\dot{\phi}^{2}}{2}+\nabla^{2} \phi+V(\phi)$. The inflaton field $\phi$ itself evolves according to a damped harmonic-oscillator-like equation of motion: $\ddot{\phi}+3 H \dot{\phi}-\nabla^{2} \phi+d V / d \phi=0$.

As the universe expands, $H$ is large and the $\dot{\phi}$ term dominates as a kind of friction term. The universe is then temporarily trapped in a slowly varying $V(\phi)$ dominated regime so that the scale factor grows exponentially. This is known as the slow roll approximation for which in the absence of spatial fluctuations becomes $\dot{\phi}=(d V / d \phi) /(3 H)$.

The biggest unknown quantity in this paradigm is the form of $V(\phi)$. The simplest form $V(\phi)=$ $(m / 2) \phi^{2}$ may be motivated by the Kahler potential in string theory, however, almost any form for 
the potential works well to describe the big bang. Recently, the determination [1] ratio of the tensor to scalar contributions to the CMB power spectrum have ruled out many of the simplest forms of the inflation generating potential. In [33] we summarized constraints on the most popular classes of models and explore some more realistic inflaton effective potentials. The constraint on standard inflationary parameters changes in the brane-world scenario. We confirm that in general the braneworld scenario increases the tensor-to-scalar ratio, thus making this paradigm less consistent with the Planck constraints. However, in the case of the lowest order $\phi^{2 / 3}$ axion monodromy there can be a slight improvement over the standard model in the case of a large number of $e$-folds $N$, particularly when compared to the Planck 15 constraints. Indeed, when BICEP2/Keck constraints are included, all monomial potentials in the brane-world scenario become disfavored compared to the standard scenario. However, for natural inflation the brane-world scenario fits the constraints better due to larger allowed values of $e$-foldings $N$ before the end of inflation in the brane-world.

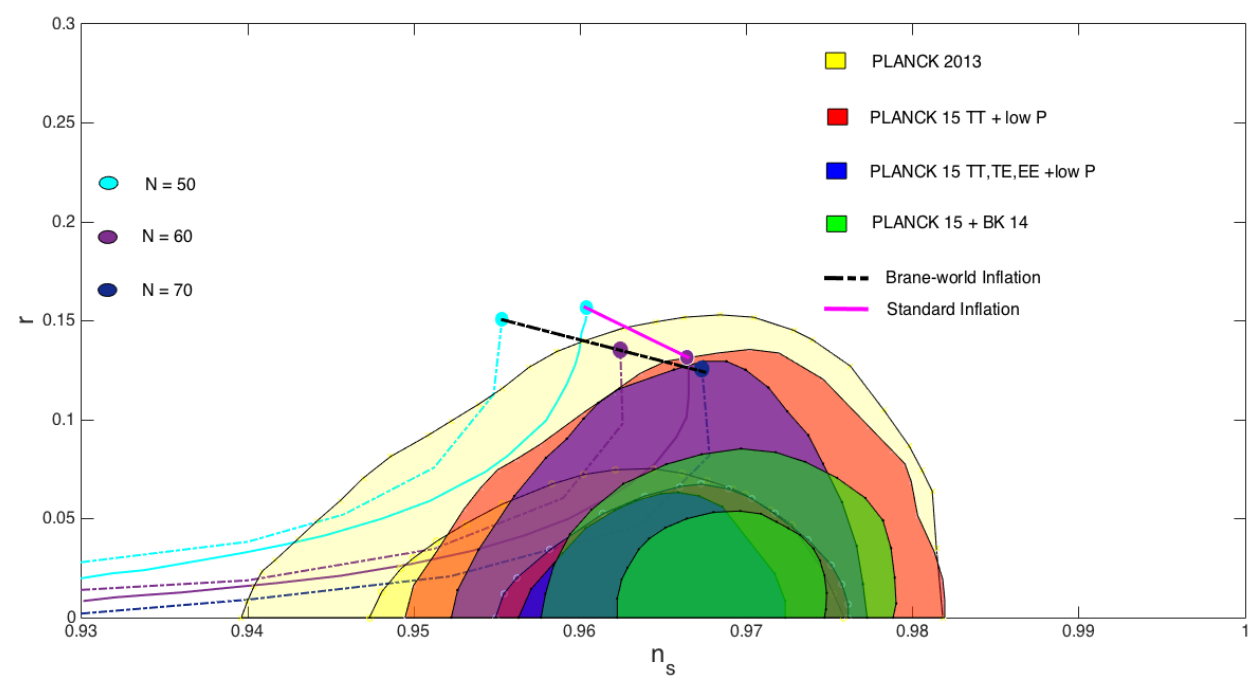

Figure 3. Effects of brane-world cosmology in the case of natural inflation compared to the standard inflation (from [33]). The legend remains the same as in previous figures. In this case, however, the dashed lines denote the range of solutions for different values of $f$ in the brane-world scenario, while the solid lines show in the standard natural inflation solution.

For the most part, BBN is unaffected by the inflation-driving potential except in the special case of quintessential inflation [35]. In Ref. [35] we looked at this intriguing attempt to reduce the inflation potential problem, the baryogenesis question, and the dark energy mystery into a single paradigm which involves non-minimal coupling between matter and gravity as the universe makes a transition from an inflation driving potential to a dark-energy producing quintessence. Big bang nucleosynthesis significantly constrains this paradigm as the non-minimal couplings lead to an excess energy density in gravity waves which alter the results of BBN. 


\section{ACKNOWLEDGMENTS}

Work at the University of Notre Dame is supported by the U.S. Department of Energy under Nuclear Theory Grant DE-FG02-95-ER40934.

\section{References}

[1] P. A. R. Ade et al., Planck XIII Collaboration, Astron. Astrophys. 594, A13 (2016)

[2] R.V. Wagoner, Ap.J. 179, 343 (1973)

[3] J. Yang, M.S. Turner, G. Steigman, D.N. Schramm, and K.A. Olive, Ap.J. 281, 493 (1984)

[4] R. A. Malaney and G. J. Mathews, G. J., Phys. Rep. 229, 145 (1993)

[5] F. Iocco et al., Phys. Rep., 47, 1 (2009)

[6] R. H. Cyburt, B. D. Fields and K. A. Olive, T.-H. Yeh, Rev. Mod. Phys. 88015004 (2016)

[7] G. J. Mathews, M. Kusakabe and T. Kajino, Int. J. Mod. Phys. E 26, 1741001 (2017)

[8] G. J. Mathews, Y. Pehlivan, T. Kajino, A. B. Balantekin and M. Kusakabe, Astrophys. J. 72710 (2011).

[9] R. H. Cyburt et al., Astrophys. J. Suppl. 189, 240 (2010)

[10] G. R. Caughlan and W. A. Fowler, At. Data Nucl. Data Tables 40, 283 (1988)

[11] C. Angulo et al., Nucl. Phys. A 656, 656 (1999)

[12] M. Foley, N. Sasankan, M. Kusakabe and G. J. Mathews, Int. J. Mod. Phys. E 26, 1741008 (2017)

[13] A. Coc and E. Vangioni, Int. J. Mod. Phys. E 26, 1741002 (2017).

[14] R. Nakamura, M.-A. Hashimoto, R. Ichimasa and K. Arai, Int. J. Mod. Phys. E 26, 1741003 (2017)

[15] P. Descouvemont et al., ADNDT, 88, 203 (2004)

[16] C. Pitrou et al., Phys. Rep. Submitted (2018)

[17] A. P. Serebrov and A. K. Fomin, Phys. Rev. C 82, 035501) (2010).

[18] G. J. Mathews T. Kajino, and T. Shima, Phys. Rev. D 71, 021302 (2005)

[19] E. Aver, K. A. Olive, and E. D. Skillman, JCAP 05, 003 (2010), JCAP 11, 017 (2013); arXiv:1503:08146.

[20] R. Cooke et al., Astrophys. J. 78131 (2014); R. Cooke et al., Astrophys. J. 830, 148 (2016)

[21] T. M. Bania, R. T. Rood and D. S. Balser, Nature 415, 54 (2002)

[22] C. Chiappini, A. Renda and F. Matteucci, Astron. Astrophys. 395, 789 (2002)

[23] E. Vangioni-Flam, K. A. Olive, B. D. Fields and M. Casse, Astrophys. J. 585, 611 (2003)

[24] M. Kusakabe, K. Kim, M.-K. Cheoun, T. Kajino, Y. Kino and G. J. Mathews, Astrophys. J. Suppl. 214, 5 (2014)

[25] M. Kusakabe, G. J. Mathews, T. Kajino and M.-K. Cheoun, Int. J. Mod. Phys. E 26, 1741004 (2017)

[26] J. Sato, T. Shimomura and M. Yamanaka, Int. J. Mod. Phys. E 26, 1741005 (2017)

[27] D. Yamazaki, M. Kusakabe, T. Kajino, G. J. Mathews and M.-K. Cheoun, Int. J. Mod. Phys. E 26, 1741006 (2017)

[28] S. Q. Hou, J. J. He, J. J. A. Parikh, D. Kahl, D. C. A. Bertulani, T. Kajino, G. J. Mathews and G. Zhao, Astrophys. J. 834, 165 (2017)

[29] P. Horava and E. Witten, Nucl. Phys. B 460, 506524 (1996); P. Horava and E. Witten, Nucl. Phys. B 475, 94114 (1996)

[30] K. Ichiki, M. Yahiro, T. Kajino, M. Orito and G. J. Mathews, Phys. Rev. D 66, 043521 (2002) 
[31] N. Sasankan, M. Gangopadhyay, M. Kusakabe and G. J. Mathews, Phys. Rev. D 95083516 (2017); Int. J. Mod. Phys. E. 26, 1740076 (2017)

[32] L. Randall and R. Sundrum, Phys. Rev. Lett. 83, 3370(1999); Phys. Rev. Lett. 83, 4690 (1999)

[33] M. R. Gangopadhyay and G. J. Mathews, JCAP, in press, arXiv: 1611.50123 hep/ph (2018)

[34] A. Starobinsky, Sov. Phys. J. Exp. Theor. Phys. Lett. 30, 682 (1979); A. Guth, Phys. Rev. D 23, 347 (1981); A. Linde, Phys. Lett. B 108, 393 (1982);

[35] M. Yahiro, G. J. Mathews, K. Ichiki, T. Kajino and M. Orito, Phys. Rev. D 65, 063502 (2002) 